\title{
Chorioretinal post-transplant lymphoproliferative disorder induced by the Epstein-Barr virus
}

\author{
P F Demols, P M Cochaux, T Velu, L Caspers-Velu
}

\begin{abstract}
Background-The Epstein-Barr virus (EBV) is responsible for the lymphoproliferative disorders observed in transplanted patients.

Methods-The case history is described of a 59 year old man with a chorioretinal lesion who had received a single lung transplant and was on immunosuppressive treatment. Immunoglobulin gene rearrangement and EBV detection by polymerase chain reaction (PCR) with semiquantification were used on the vitreous material.

Results-A proliferation of B lymphocytes with a monoclonal subpopulation was found by PCR on the vitreous sample. The large amounts of EBV genomes found in the vitreous suggest that $E B V$ was the cause of the lymphoproliferation. Healing of the lesion was achieved by a decrease in immunosuppressive treatment and the use of nucleotide analogues.

Conclusion-The diagnosis of ocular posttransplant lymphoproliferative disorder (PTLD) can be made by PCR on vitreous material. Early diagnosis and treatment can lead to regression of limited monoclonal lesions.

(Br f Ophthalmol 2001;85:93-95)
\end{abstract}

The use of immunosuppressive agents following transplantation has already saved many lives, but the incidence of neoplasms has increased. ${ }^{1}$ Lymphomas which occur in allograft recipients are mainly of $\mathrm{B}$ cell origin. Post-transplant lymphoproliferative disorders (PTLD) include a number of diseases ranging from infectious mononucleosis-like syndrome to malignant lymphoma.

We report a case of chorioretinal PTLD in whom large amounts of Epstein-Barr virus (EBV) DNA were detected by semiquantitative polymerase chain reaction (PCR) in the vitreous.

\section{Case report}

A 59 year old man received a single lung transplant for emphysema in December 1992 and was treated with immunosuppressive drugs (cyclosporin, azathioprine, and methylprednisolone). He had already needed treatment for Pneumocystis carinii and cytomegalovirus (CMV) lung infections, relapsing herpes zoster, and episodes of positive CMV antigenaemia.

In November 1994 the patient presented to our clinic complaining of floaters and decreased vision in the right eye for 2 days. Because of a recent relapse of CMV antigenaemia, the immunosuppressive treatment had recently been decreased (cyclosporin from $300 \mathrm{mg}$ to $250 \mathrm{mg}$ daily, azathioprine $50 \mathrm{mg}$ daily was discontinued, and methylprednisolone remained at $8 \mathrm{mg}$ daily). Intravenous ganciclovir had been administered $(350 \mathrm{mg} /$ day 5 days a week) for 5 days. At first examination visual acuity was $20 / 35$ in the right eye and $20 / 15$ in the left. There was $1+$ anterior cells, a $1+$ vitreous haze, and a thick homogeneous yellow-white chorioretinal lesion along the superior temporal arcade extending to the posterior pole with an arteriovenous thrombosis of the superotemporal branch with minimal haemorrhages.

The diagnosis was thought to be atypical CMV retinitis or toxoplasmic retinochoroiditis and treatment with intravenous ganciclovir was continued. Two days later the dose of cyclosporin was further reduced. After 2 weeks the chorioretinal lesion extended to the macular region with the appearance of a few haemorrhages and aggravation of the vitreous haze (Fig 1A). Many granulomatous keratic precipitates appeared and the visual acuity dropped to finger counting. The first molecular study by PCR did not reveal CMV, herpes simplex virus (HSV), varicella zoster virus (VZV), or toxoplasmosis in the vitreous. A weak PCR positivity was present for Pneumocystis carinii in the vitreous but the clear positivity in the serum suggested contamination of the vitreous with blood during vitrectomy. As expected, a trial of oral trimethoprim and sulfamethoxazole did not improve the ocular lesion and confirmed this hypothesis. Semi-quantitative PCR revealed the presence of a large number of EBV genomes in the vitreous material (Fig 2).

Polymerase chain reaction of the EBV genome was performed using TC60 and TC61 primers derived, as previously described, ${ }^{2}$ from the Bam HI W region, giving an amplified fragment of 122 base pairs. The PCR mixture contained $200 \mu \mathrm{M}$ dNTP, $2.5 \mathrm{mM} \mathrm{MgCl}_{2}$, $50 \mathrm{mM} \mathrm{KCl}, 10 \mathrm{mM}$ TRIS HCl (pH 8.3), $0.1 \mathrm{mg} / \mathrm{ml}$ gelatin, $2.5 \mathrm{U}$ Taq DNA polymerase, and $1 \mu \mathrm{g}$ of each primer for a total reaction volume of $50 \mu \mathrm{l}$. As a control for gene amplification, primers derived from the phenylalanine hydroxylase gene, giving an amplified product of 260 base pairs, were used (5A and 5B). ${ }^{3}$ Reactions were incubated in a Biometra Trioblock cycler for 35 cycles (48 seconds at $93^{\circ} \mathrm{C}, 48$ seconds at $53^{\circ} \mathrm{C}, 90$ seconds at $72^{\circ} \mathrm{C}$; last extension cycle of 6 minutes). The PCR products were analysed by agarose gel stained with ethidium bromide and by transfer and hybridisation with a ${ }^{32} \mathrm{P}$ labelled probe (TC62 probe specific for the EBV Bam HI W fragment). ${ }^{2}$ The semiquantification was assessed by comparison of the sample PCR 


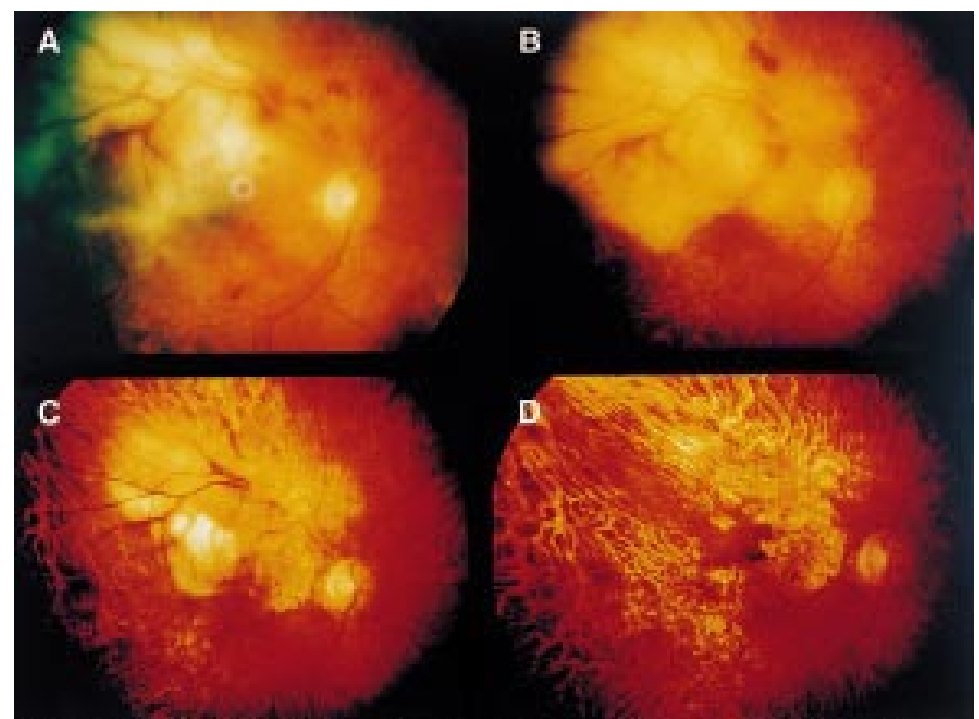

Figure 1 (A) Chorioretinal yellow-white lesion extending towards the macula with arterial and venous thrombosis of the superotemporal arcade. (B-D) Progressive healing of the chorioretina over 6 months. (B) Fanuary 1995, (C) March 1995, (D) Fuly 1995.

product with amplified dilutions of decreasing amounts of EBV control plasmid DNA mixed with DNA from uninfected cells. The plasmid pBA-W containing the Bam HI fragment from the B95-8 EBV genome was kindly provided by A Sergent (CNRS, Lyons, France). ${ }^{4}$

Further molecular study with PCR revealed rearrangement of a clonal immunoglobulin

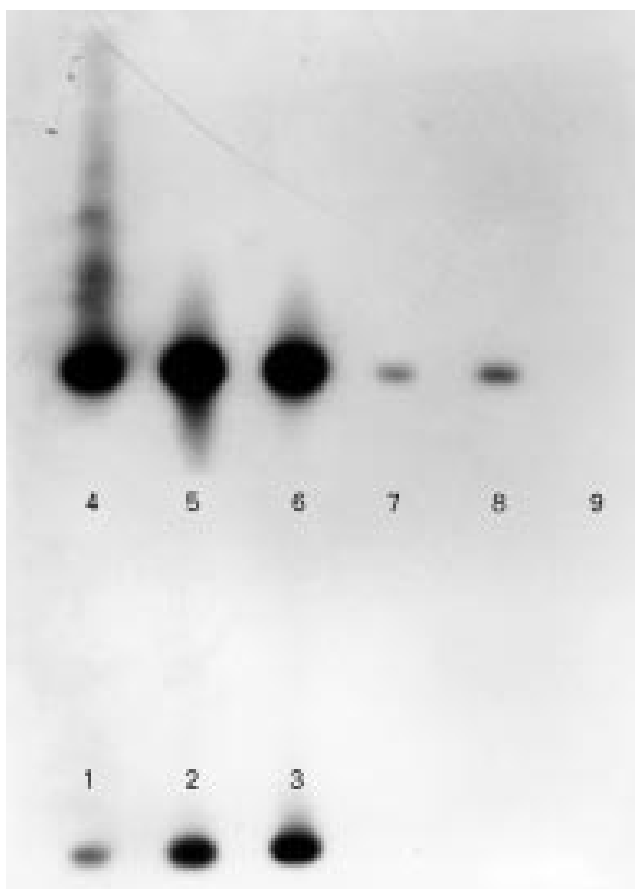

Figure 2 Semiquantitative EBV PCR. Lanes 1-4: control EBV plasmid dilutions $10^{-9}, 10^{-8}, 10^{-7}$, and $10^{-6}(0.1,1,10$, and $100 \mathrm{EBV}$ genomes 1000 cells, respectively); lanes 5 and 6: vitreous material; lane 7: blood sample from the same patient; lanes 8 and 9: blood samples from another patient, the second one being EBV negative. The amount of $E B V$ genomes is estimated to be lower than $10^{-9}$ in the blood and higher than $10^{-6}$ in the vitreous. The amount of $E B V$ genomes in the vitreous material appears to be at least 1000 times higher than in the blood, and is probably largely underestimated since the ethidium bromide staining of the control amplification (see Methods) showed that there were only a few cells in the vitreous material compared with the blood sample. gene at the heavy chain locus, showing that a monoclonal cell population had emerged among the EBV positive proliferating $\mathrm{B}$ lymphocytes.

Serological tests showed an increase in the EBV viral capsid antigen (VCA) antibodies (IgG $1 / 320 \rightarrow 1 / 1280$ ) between August and October 1994 which strongly supported the diagnosis of chorioretinal EBV induced monoclonal PTLD.

EBV genomes decreased in the vitreous fluid with intravitreous administration of ganciclovir and foscavir in association with intravenous ganciclovir, although the lesion continued to grow. This antiviral treatment was then replaced with intravenous aciclovir ( $1 \mathrm{~g}$ twice daily) for 3 weeks from 26 December 1994 followed by oral administration ( $800 \mathrm{mg}$ three times daily) because of chronic renal failure. This led to clinical improvement of the lesion until total scarring was achieved in June 1995, 6 months after the start of treatment (Fig 1B-D). There has been no ocular recurrence to date.

\section{Discussion}

EBV induced lymphoproliferative disorders are observed in both iatrogenically and congenitally immunosuppressed patients. ${ }^{5}$ The discovery of significant amounts of EBV genomes in the immunodeficient tissues of patients with lymphoproliferative disorders suggests that EBV is the responsible agent.

The pathogenesis of PTLD is complex and multifactorial. It is caused by a breakdown in the balance between $\mathrm{B}$ cell stimulation and the anti-EBV immune response. B lymphocyte proliferation is induced by chronic antigenic stimulation from the allograft, by the presence of an oncogene in the genome of the EBV, and by the degree of immunosuppression. ${ }^{15-7}$ When there is an imbalance, polyclonal B lymphocyte proliferation occurs that may develop into an oligoclonal or monoclonal lymphoma. ${ }^{589}$ Some clones are susceptible to genetic modifications-for example, oncogene or tumour suppressor gene alterations which result in the development of a true lymphoma. ${ }^{9}$

The clonal state is not the only criterion for malignancy. In the case presented here, a monoclonal state occurred with no malignancy. The major risk factors for developing PTLD are the intensity, type, and duration of immunosuppressive therapy. ${ }^{2}{ }^{3}$ Non-clonal PTLD and a subpopulation of clonal PTLD regress when the dose of immunosuppressive treatment is decreased, with or without aciclovir. ${ }^{56}$

Clinical, morphological, immunological, and cytogenetic analysis enables the therapeutic response to be predicted. ${ }^{5610}$ Patients with stage I PTLD (lymphoid hyperplasia) respond to treatment with aciclovir, often without a reduction in the dose of immunosuppressive treatment, thus avoiding any risk of graft rejection. ${ }^{510}$ At the other extreme, stage III PTLD (true lymphoma) progresses in spite of a decrease in immunosuppressive treatment, with or without aciclovir. ${ }^{510}$ These lesions require standard antineoplasic treatment (chemotherapy, radiotherapy, surgery) and the discontinuation of immunosuppressive drugs if 
possible. ${ }^{5}{ }^{10}$ Aciclovir has been shown to be ineffective when the tumour is monoclonal, probably because it consists of latent infected $\mathrm{B}$ cells which no longer depend on EBV replication to proliferate but have acquired a malignant autonomous growth potential. ${ }^{510}$ Nevertheless, an early clonal subpopulation (stage II PTLD) may respond to aciclovir treatment associated with a reduction in immunosuppression if the autonomous growth potential has not yet been reached. ${ }^{10}$

Some cases of chorioretinitis with serologically determined acute EBV infection have been observed, ${ }^{11-13}$ but without solid evidence of their cause. A few cases of intraocular lymphoproliferation associated with AIDS have been reported, and EBV has recently been found by in situ hybridisation in a retinal biopsy specimen of an HIV positive patient with multifocal chorioretinitis. ${ }^{14} \mathrm{EBV}$ has been suspected but its presence has not been confirmed in another four cases of intraocular lymphoproliferation following transplantation. ${ }^{15-18}$

The case reported here is the first documented case of chorioretinal PTLD in whom a large number of EBV genome copies have been found in vitreous cells by PCR semiquantification associated with monoclonal proliferation. This case also confirms that a monoclonal proliferation can regress with decreased immunosuppressive therapy associated with nucleotide analogues. It also shows that the diagnosis of intraocular EBV induced PTLD can be made from the vitreous alone using a PCR amplification technique which permits semiquantification of the virus.

Early detection and treatment are essential for the regression of limited monoclonal lesions. Identification of patients who are at high risk for this kind of complication might lead to a decrease in morbidity and mortality in this population.
1. Penn I. Cancers complicating organ transplantation. N Engl f Med 1990;323:1767-9.

2 Saito I, Servenius B, Compton T, et al. Detection of Epstein-Barr virus DNA by polymerase chain reaction in blood and tissue biopsies from patients with Sjogren's syndrome. F Exp Med 1989;169:2191-8.

3 Okano Y, Wang T, Eisensmith RC, et al. Missense mutations associated with RFLP haplotypes 1 and 4 of the human phenylalanine hydroxylase gene. Am f Hum Genet 1990;46: $18-25$.

4 Jones MD, Griffin BE. Clustered repeat sequences in the genome of Epstein-Barr virus. Nucleic Acids Res 1983;11: 3919-37.

5 Hanto DW, Gajl-Peczalska KJ, Frizzera G, et al. EpsteinBarr virus (EBV) induced polyclonal and monoclonal B-cell lymphoproliferative diseases occurring after renal transplantation. Ann Surg 1983;198:356-69.

6 Nalesnik MA, Jaffe R, Starzl TE, et al. The pathology of posttransplant lymphoproliferative disorders occurring in the setting of cyclosporine A-prednisone immunosuppression. Am $\mathcal{F}$ Pathol 1988;133:173-92.

7 Cockfield SM, Preiksaitis JK, Jewell LD, et al. Posttransplant lymphoproliferative disorder in renal allograft recipients. Transplantation 1993;56:88-96.

8 Cleary ML, Warnke R, Sklar J. Monoclonality of lymphoproliferative lesions in cardiac-transplant recipients. N Engl f Med 1984;310:477-82.

9 Knowles DM, Cesarman E, Chadburn A, et al. Correlative morphologic and molecular genetic analysis demonstrates three distinct categories of posttransplantation lymphoproliferative disorders. Blood 1995;85:552-65.

10 Hanto DW, Frizzera G, Gajl-Peczalska KJ, et al. Acyclovir therapy of Epstein-Barr virus-induced posttransplant lymphoproliferative diseases. Transplant Proc 1985;17:89-92.

11 Bonamour G, Pommier ML. Manifestations choriorétiniennes dans la mononucléose infectieuse. Bull Soc Ophtalmol Fr 1968;68:52-4.

12 Kelly SP, Rosenthal AR, Nicholson KG, et al. Retinochoroiditis in acute Epstein-Barr virus infection. $\mathrm{BrF} \mathrm{Oph-}$ thalmol 1989;73:1002-3.

13 Tiedeman JS. Epstein-Barr viral antibodies in multifocal choroiditis and panuveitis. Am $\mathcal{f}$ Ophthalmol 1987;103: 659-63.

14 Mittra RA, Pulido JS, Hanson GA, et al. Primary ocular Epstein-Barr virus-associated non-Hodgkin's lymphoma in a patient with AIDS. Retina 1999;19:45-50.

15 Johnson BL. Intraocular and central nervous system lymphoma in a cardiac transplant recipient. Ophthalmology 1992;99:987-92.

16 Kheterpal S, Kirkby GR, Neuberger JM, et al. Intraocular lymphoma after liver transplantation. Am $\mathcal{f}$ Ophthalmol 1993;116:507-8

17 Robinson R, Murray PI, Willshaw HE, et al. Primary ocular posttransplant lymphoproliferative disease. $\mathcal{F}$ Pediatr $O p h$ thalmol Strabismus 1995;32:393-4.

18 Ziemianski MC, Godfrey WA, Lee KY, et al. Lymphoma of the vitreous associated with renal transplantation and immunosuppressive therapy. Ophthalmology 1980;87:596601 . 